\title{
Article \\ Is There a Difference in Clinical Features, Microbiological Epidemiology and Effective Empiric Antimicrobial Therapy Comparing Healthcare-Associated and Community-Acquired Vertebral Osteomyelitis?
}

\author{
Siegmund Lang ${ }^{1, * \mathbb{D}}$, Astrid Frömming ${ }^{1}$, Nike Walter ${ }^{1}$, Viola Freigang ${ }^{1}$, Carsten Neumann ${ }^{1}$, Markus Loibl ${ }^{1,2}$, \\ Martin Ehrenschwender ${ }^{3}$, Volker Alt ${ }^{1} \mathbb{D}$ and Markus Rupp ${ }^{1}$ \\ 1 Department of Trauma Surgery, University Hospital Regensburg, Franz-Josef-Strauss-Allee 11, \\ 93053 Regensburg, Germany; astrid.froemming@t-online.de (A.F.); \\ Nike.Walter@klinik.uni-regensburg.de (N.W.); viola.freigang@klinik.uni-regensburg.de (V.F.); \\ carsten.neumann@ukr.de (C.N.); markus.loibl@kws.ch (M.L.); volker.alt@ukr.de (V.A.); \\ Markus.Rupp@klinik.uni-regensburg.de (M.R.) \\ 2 Department of Spine Surgery, Schulthess Clinic, Lenghalde 2, 8008 Zurich, Switzerland \\ 3 Institute of Clinical Microbiology and Hygiene, University Hospital Regensburg, Franz-Josef-Strauss-Allee 11, \\ 93053 Regensburg, Germany; Martin.Ehrenschwender@barmherzige-regensburg.de \\ check for \\ updates \\ * Correspondence: Siegmund.lang@klinik.uni-regensburg.de; Tel.: +49-9419446799
}

Citation: Lang, S.; Frömming, A.; Walter, N.; Freigang, V.; Neumann, C.; Loibl, M.; Ehrenschwender, M.; Alt, V.; Rupp, M. Is There a Difference in Clinical Features, Microbiological Epidemiology and Effective Empiric Antimicrobial Therapy Comparing

Healthcare-Associated and

Community-Acquired Vertebral Osteomyelitis? Antibiotics 2021, 10, 1410. https://doi.org/10.3390/ antibiotics 10111410

Academic Editors: Gianluigi Franci and Giovanni Boccia

Received: 18 October 2021

Accepted: 15 November 2021

Published: 18 November 2021

Publisher's Note: MDPI stays neutral with regard to jurisdictional claims in published maps and institutional affiliations.

Copyright: (C) 2021 by the authors. Licensee MDPI, Basel, Switzerland. This article is an open access article distributed under the terms and conditions of the Creative Commons Attribution (CC BY) license (https:// creativecommons.org/licenses/by/ $4.0 /)$.

\begin{abstract}
Background: Empiric antibiotic therapy for suspected vertebral osteomyelitis (VO) should be initiated immediately in severely ill patients, and might be necessary for culture-negative VO. The current study aimed to identify differences between community-acquired (CA) and healthcareassociated (HA) VO in terms of clinical presentation, causative pathogens, and antibiotic susceptibility. Methods: Cases of adult patients with VO treated at a German university orthopaedic trauma center between 2000 and 2020 were retrospectively reviewed. Patient history was used to distinguish between CA and HA VO. Susceptibility of antibiotic regimens was assessed based on antibiograms of the isolated pathogens. Results: A total of 155 patients (with a male to female ratio of 1.3; and a mean age of $66.1 \pm 12.4$ years) with VO were identified. In $74(47.7 \%)$ patients, infections were deemed healthcare-associated. The most frequently identified pathogens were Staphylococcus aureus (HAVO: 51.2\%; CAVO: 46.8\%), and Coagulase-negative Staphylococci (CoNS, HAVO: 31.7\%; CAVO: 21.3\%). Antibiograms of 45 patients (HAVO: $n=22$; CAVO: $n=23$ ) were evaluated. Significantly more methicillin-resistant isolates, mainly CoNS, were found in the HAVO cohort (27.3\%). The highest rate of resistance was found for cefazolin (HAVO: 45.5\%; CAVO: 26.1\%). Significantly higher rates of resistances were seen in the HAVO cohort for mono-therapies with meropenem (36.4\%), piperacillintazobactam $(31.8 \%)$, ceftriaxone $(27.3 \%)$, and co-amoxiclav $(31.8 \%)$. The broadest antimicrobial coverage was achieved with either a combination of piperacillin-tazobactam + vancomycin (CAVO: 100.0\%; HAVO: 90.9\%) or meropenem + vancomycin (CAVO: 100.0\%; HAVO: 95.5\%). Conclusion: Healthcare association is common in VO. The susceptibility pattern of underlying pathogens differs from CAVO. When choosing an empiric antibiotic, combination therapy must be considered.
\end{abstract}

Keywords: vertebral osteomyelitis; healthcare-associated infections; antimicrobial resistance; epidemiology; spine; coagulase-negative staphylococci; systemic antibiotic therapy; treatment

\section{Introduction}

Hematogenous pyogenic vertebral osteomyelitis (VO) is one of the most frequent manifestation of hematogenous osteomyelitis in adults with an estimated incidence of 2.4 cases per 100,000 people in European countries [1,2]. An increasing incidence of VO has been reported, which is mainly due to two reasons. First, susceptible populations with a history of an increasing number of previous spine surgeries. Second, the improved 
accuracy in diagnosis results in earlier and more frequent detection of VO [3]. With increasing age, and thus related higher prevalence of chronic diseases of the European population, the incidence of vertebral osteomyelitis is expected to further increase [4,5]. The most common causative pathogen of VO in Europe is Staphylococcus aureus (S. aureus). In more than $50 \%$ of the culture-positive cases, S. aureus is responsible for VO, followed by up to 11-25\% of VO caused by Gram-negative pathogens [6]. Insidious and indolent courses of $\mathrm{VO}$ often result in delayed diagnosis, which potentially leads to high morbidity and mortality [7]. Predisposing risk factors for VO are previous spine surgery, a distant infectious focus, diabetes mellitus, advanced age, intravenous drug use, HIV infection, immunosuppression, oncologic history, renal failure, rheumatological diseases, and liver cirrhosis [8]. Recently, healthcare-associated vertebral osteomyelitis (HAVO) has been reported to make a relevant share of all VO cases. HAVO reportedly lead to higher rates of mortality and higher recurrence of infection compared to community-acquired vertebral osteomyelitis (CAVO) [9]. Further, HAVO has been associated with prolonged antibiotic therapy and a decreased functional status $[10,11]$. One main source for HAVO is (intravascular) catheter-related and device-related bloodstream infection. Up to $34.0 \%$ of HAVO coagulase-negative staphylococci could be isolated from blood cultures [12].

In both CAVO and HAVO, empiric antibiotic therapy is often necessary. Indications range from severe or progressive neurologic deficits, hemodynamic instability, and culturenegative VO $[6,13]$. To achieve best possible empirical antibiotic coverage, susceptibility of the most likely pathogens being causative for VO must be considered.

Therefore, the present study aims to answer the following questions. (1) What are the differences of HAVO and CAVO in terms of clinical presentation and treatment characteristics? (2) What are the causative pathogens in HAVO and CAVO? (3) What is the best possible empirical antibiotic therapy in both HAVO and CAVO? (4) Is there any difference of antibiotic susceptibility comparing pathogens evidenced in HAVO and CAVO?

\section{Results}

\subsection{Patient Characteristics, Clinical Presentation, and Treatment (1)}

Of the 253 patients with ICD-10 codes of interest, 155 were identified as having hematogenous vertebral osteomyelitis and met the inclusion criteria (Figure 1). In $n=65$ cases, the above-mentioned criteria for the diagnosis of pyogenic VO were not fulfilled based on the medical records and, thus, they were excluded (incorrect ICD-10 coding). Table 1 displays the baseline data of all included patients and a differentiation between CAVO and HAVO-classified cases.

Overall, $n=88(56.8 \%)$ of the patients were male and $67(43.2 \%)$ female (male to female ratio $=1.3)$. The mean age was $66.1 \pm 12.4$ years. The mean body-mass index (BMI) was $29.0 \pm 8.2 \mathrm{~kg} / \mathrm{m}^{2}$. Most patients had comorbidities, resulting in a median Charlson comorbidity index (CCI) of 1 point (range 0-8 points; mean: $1.8 \pm 1.8$ points). Fifty-five (35.5\%) patients showed a CCI between 2, 3, and 28 (18.1\%), i.e., a CCI higher than three points. In $48(31.0 \%)$ cases, the duration of symptoms could be determined on the basis of the medial records. The mean duration of symptoms was $63.7 \pm 82.2$ days. One-hundred and seven patients $(69.0 \%)$ reported back pain at admission and $n=18$ $(11.6 \%)$ suffered from neurological deficits. Twenty-five $(16.1 \%)$ patients presented with fever (body temperature $>38.0^{\circ} \mathrm{C}$ ). The mean first CRP (C-reactive protein) value was $132.4 \pm 104.1$ (range 3.0-498.0) $\mathrm{mg} / \mathrm{L}$ and $n=66(42.6 \%$ ) patients had a CRP of $>100 \mathrm{mg} / \mathrm{L}$.

There was no statistically significant difference in the mean CRP value between the CAVO $(135.6 \pm 111.7 \mathrm{mg} / \mathrm{L})$ and the HAVO $(128.5 \pm 94.8 \mathrm{mg} / \mathrm{L})$ cohort $(p=0.709)$. The mean leucocyte count at admission was $11.0 \pm 4.6$ (range 2.9-29.6) $\times 10^{9} / \mathrm{L}$. Again, there was no difference in the leucocyte counts between the two cohorts $(p=0.094)$. During hospitalization, 34 (21.9\%) patients developed septic symptoms. Twenty patients died during the hospitalization (in-hospital mortality rate $=12.9 \%$ ). The most frequently documented reason for death was (septic) multi-organ failure $(n=10)$ followed by cardiac reasons $(n=4)$. In two cases, the reason for death was not documented in detail. There 
was no statistically significant difference in the number of in-hospital deaths between the CAVO $(n=9)$ and HAVO $(n=11)$ group $(p=0.485)$. The documented causes for in-hospital deaths are given in Table 2.

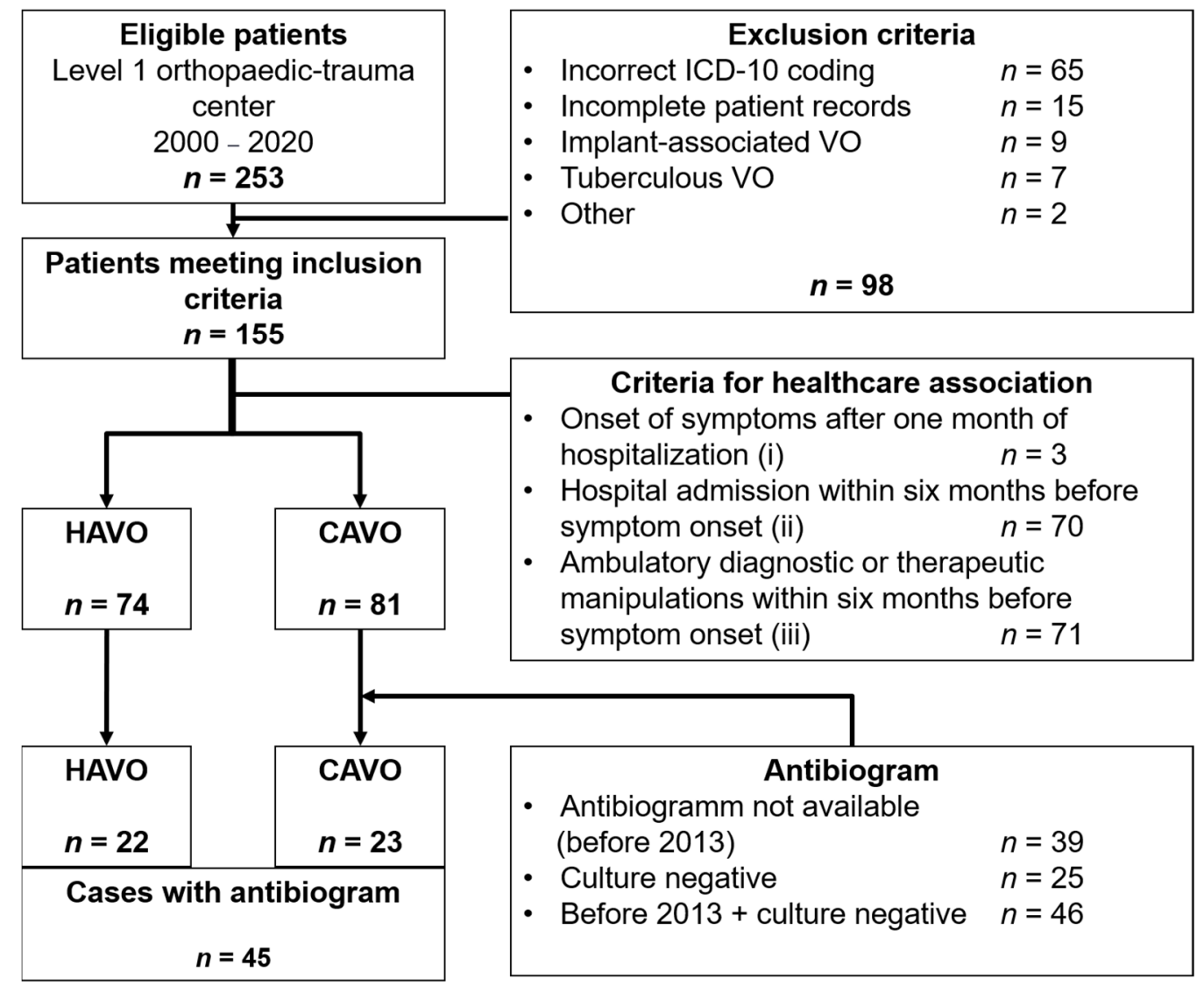

Figure 1. Flowchart of patients included in the study. Abbreviations: HAVO: Healthcare-associated vertebral osteomyelitis. CAVO: Community-acquired osteomyelitis.

Table 1. Baseline characteristics of all patients and the two cohorts consisting of CAVO and HAVO patients. Data on age, BMI, duration of symptoms, and hospitalization is given as mean values \pm standard deviation. CCI points are given as median with range and mean \pm standard deviation. All other parameters are displayed numerically and as a percentage of the respective cohort.

\begin{tabular}{cccc}
\hline Characteristic & All $(\boldsymbol{n}=\mathbf{1 5 5})$ & CAVO $(\boldsymbol{n}=\mathbf{8 1})$ & HAVO $(\boldsymbol{n}=\mathbf{7 4})$ \\
\hline Demographic data & & & \\
Sex (male) & $88(56.8 \%)$ & $41(50.6 \%)$ & $47(63.5 \%)$ \\
Age (years) & $66.1 \pm 12.4$ & $66.6 \pm 12.9$ & $65.6 \pm 11.9$ \\
BMI (kg/m $\left.{ }^{2}\right)$ & $29.0 \pm 8.2$ & $29.9 \pm 9.0$ & $28.4 \pm 7.7$ \\
CCI & $1[0-8](1.8 \pm 1.8)$ & $1[0-7](1.5 \pm 1.6)$ & $2[0-8](2.1 \pm 1.9)$ \\
Location & & & $4(5.4 \%)$ \\
Cervical spine & $12(7.7 \%)$ & $8(9.9 \%)$ & $23(31.1 \%)$ \\
Thoracic spine & $57(36.8 \%)$ & $34(42.0 \%)$ & $39(52.7 \%)$ \\
Lumbar spine & $74(47.7 \%)$ & $35(43.2 \%)$ & $8(10.8 \%)$ \\
Multifocal & $12(7.7 \%)$ & $4(4.9 \%)$ & $69.9 \pm 96.9$ \\
Duration of symptoms (days) & $63.7 \pm 82.2$ & $56.9 \pm 64.2$ & $40.6 \pm 46.5$ \\
Hospitalization (days) & $36.3 \pm 36.3$ & $92.3 \pm 23.0$ & $11(14.9 \%)$ \\
In-hospital deaths & $20(12.9 \%)$ & $34(41.1 \%)$ & $33(44.6 \%)$ \\
Microbiologic results & & $23(28.4 \%)$ & $22(29.7 \%)$ \\
Culture-negative & $67(43.2 \%)$ & $35(43.2 \%)$ & $35(47.3 \%)$ \\
Antibiogram available & $45(29.0 \%)$ & & \\
Positive blood culture & $70(45.2 \%)$ & & \\
\hline
\end{tabular}


Table 2. Causes of in-hospital deaths in the CAVO and the HAVO cohort in total numbers and percentages of cases. The age at the time of death is given separately for each case and as mean \pm standard deviation for the respective cohort.

\begin{tabular}{|c|c|c|c|c|c|c|}
\hline \multirow[b]{2}{*}{ Reason } & \multicolumn{3}{|c|}{ CAVO $(n=9)$} & \multicolumn{3}{|c|}{ HAVO $(n=11)$} \\
\hline & $n$ & $\%$ & Age [years] & $n$ & $\%$ & Age [years] \\
\hline Cardiovascular arrest & 2 & 22.2 & 65:74 & 2 & 18.2 & 66:79 \\
\hline Multi-organ failure & 3 & 33.3 & $69 ; 75 ; 77$ & 4 & 36.4 & $50 ; 71 ; 71 ; 74$ \\
\hline Sepsis (with multi-organ failure) & 3 & 33.3 & $74 ; 83 ; 83$ & - & - & - \\
\hline Drug intoxication & 1 & 11.1 & 32 & - & - & - \\
\hline Respiratory insufficiency & - & - & - & 1 & 9.1 & 69 \\
\hline Graft versus host disease & - & - & - & 1 & 9.1 & 67 \\
\hline Fungal pneumonia & - & - & - & 1 & 9.1 & 63 \\
\hline \multirow[t]{2}{*}{ Not documented } & - & - & - & 2 & 18.2 & $79 ; 85$ \\
\hline & \multicolumn{2}{|c|}{ Mean age } & $70.1 \pm 15.4$ & \multicolumn{2}{|c|}{ Mean age } & $70.4 \pm 9.4$ \\
\hline
\end{tabular}

$\mathrm{VO}$ was diagnosed at the cervical spine in $12(7.7 \%)$ cases, at the thoracic spine in 57 $(36.8 \%)$ and at the lumbar spine in $74(47.7 \%)$ cases (Table 1). A multifocal location of $\mathrm{VO}$ was seen in $12(7.7 \%)$ patients.

According to the patient records, $\mathrm{VO}$ was classified as healthcare-associated (HA) in $74(47.7 \%)$ of the cases and as community-acquired (CA) in $81(52.3 \%)$. In all the HAVO-classified cases, the criterion (iii) was applicable, and, in 70 (94.6\%) cases, a hospital admission within six months before symptom onset (ii) was documented.

There was no statistically significant difference in the age $(p=0.624)$, sex $(p=0.105)$, BMI $(p=0.390)$, and the mean CCI $(p=0.378)$ between CAVO and HAVO cases. There were no statistically significant differences in the incidence of fever $(p=0.367)$, b-symptoms ( $p=0.174)$, or sepsis $(p=0.386)$ between the CAVO and HAVO cohort. The distribution of the localization of VO did not statistically or significantly differ between the CAVO and HAVO group $(p=0.195)$.

Magnetic resonance imaging (MRI was conducted in 98 (63.2\%) cases and a computerized tomography (CT) scan in $95(61.3 \%)$ cases. In those, MRI revealed clear signs of VO in $99.0 \%$ of the scans and CT showed typical bone destruction in $98.9 \%$ of the scans. There was no significant difference in the number of cases with CT or MRI between the CAVO (CT $n=49(60.5 \%)$ and MRI $n=51(64.2 \%))$ and the HAVO cohort (CT $n=46(62.2 \%)$ and MRI $n=46(62.2 \%)) p=0.322$ and $p=0.347)$. In the CAVO cohort, CT revealed signs of VO in $98.0 \%$ and MRI in $98.1 \%$. In the HAVO cohort, both CT and MRI showed a sensitivity of $100.0 \%$. In $n=42$ (27.1\%; CAVO; $n=25$ (30.9\%); HAVO; $n=17(23.0 \%) ; p=0.193)$ cases, a psoas abscess was identified, and paravertebral abscess formations were reported in $n=30$ $(19.4 \% ; \mathrm{CAVO} ; n=20(24.7 \%) ; \mathrm{HAVO} ; n=10(13.5 \%) ; p=0.064)$. An epidural abscess was found in $n=16(10.3 \%$; CAVO; $n=7(8.6 \%) ; \mathrm{HAVO} ; n=9(12.2 \%) ; p=0.321)$ cases.

Of all patients, 97 (62.6\%) were treated surgically. In $28(18.1 \%)$ cases, a CT-graphically guided abscess drainage was applied. Although HAVO patients showed a trend of longer duration of symptoms ( $59.6 \pm 64.2$ vs. $69.9 \pm 96.9$ days) and longer hospitalization time $(32.3 \pm 23.0$ vs. $40.6 \pm 46.5$ days), those differences were not statistically significant $(p=0.588$ and 0.157$)$.

Ninety-eight patients $(63.2 \%)$ received an empiric antibiotic treatment, prior to the identification of a pathogen. Flucloxacillin was administered most frequently in $24(24.5 \%)$ cases, followed by a cephalosporin (ceftriaxon, cefazolin, or ceftazidim) in 18 cases (18.4\%), and vancomycin $(n=13 ; 13.3 \%)$ and clindamycin in 7 cases $(7.1 \%)$. Combined antibiosis was used in 70 cases $(45.2 \%)$ and rifampicin was most frequently used for a combination in 24 cases (34.3\%). From 45 cases, in which the antibiogram could be evaluated retrospectively, 26 patients received an empiric antibiotic treatment. The empiric antibiosis had to be changed accordingly to the antibiogram in 11 (42.3\%) cases, whereas in $15(57.7 \%)$ cases, the empiric antibiosis was found to be adequate. 


\subsection{Aetiology and Microbiological Pattern (2)}

The most common isolate in the whole cohort was S. aureus (48.9\%), coagulasenegative Staphylococci (CoNS) (26.1\%), Enterobacteriales (10.2\%), and Streptococcus species $(6.8 \%)$. In $34(42.0 \%)$ of the CAVO cases and in 33 cases $(44.6 \%)$ of the HAVO patients, the infection was culture-negative. No polymicrobial infection was documented. Isolated microorganisms of both cohorts are presented in Figure 2. S. aureus was the most frequently detected pathogen in both cohorts ( $46.8 \%$ CAVO vs. $51.2 \%$ HAVO). This was followed by CoNS $(21.3 \%$ CAVO vs. $31.7 \%$ HAVO; $p=0.440)$. Table 3 shows the distribution of causative pathogens between the HAVO and CAVO cohort with antibiograms; S. aureus was the most frequently detected pathogen (HAVO $45.5 \%$; CAVO $47.8 \%$ ). In the CAVO cohort, the second most frequently detected pathogens were Enterobactericales $(21.7 \%)$, whereas in the HAVO cohort, CoNS were second most frequent (27.3\%). However, this difference was not statistically significant $(p=0.342)$. Five of the six CoNS isolates and one S. aureus (MRSA) isolate in the HAVO group were methicillin-resistant (27.3\%). In contrast, no methicillin-resistant isolates were found in the CAVO cohort $(p<0.01)$. In sum, $82.2 \%$ (CAVO: 73.9\%; HAVO: 90.1\%) of pathogens were classified as Gram-positive and $17.8 \%$ (CAVO: 26.1\%; HAVO: 9.1\%) were Gram-negative. No statistically significant difference in this distribution between the CAVO and the HAVO cohort was detected $(p=0.135)$.

Table 3. Isolated microorganisms in CAVO and HAVO patients with antibiogram $(n=45)$.

\begin{tabular}{ccc}
\hline Pathogen & CAVO $(\boldsymbol{n = 2 3 )}$ & HAVO $(\boldsymbol{n}=\mathbf{2 2})$ \\
\hline Staphylococcus aureus & $11(47.8 \%)$ & $10(45.5 \%)$ \\
Coagulase-negative staphylococci & $2(8.7 \%)$ & $6(27.3 \%)$ \\
Streptococcus species & $2(8.7 \%)$ & $2(9.1 \%)$ \\
Enterobactericales & $5(21.7 \%)$ & $2(9.1 \%)$ \\
Enterococcus species & $1(4.3 \%)($ E. faecium $)$ & $2(9.1 \%)$ \\
Other & 2(8.7\%) & - \\
& (Haemophilus parainfluenzae; & \\
\hline
\end{tabular}

\section{(a) Community-acquired VO $(n=47)$}

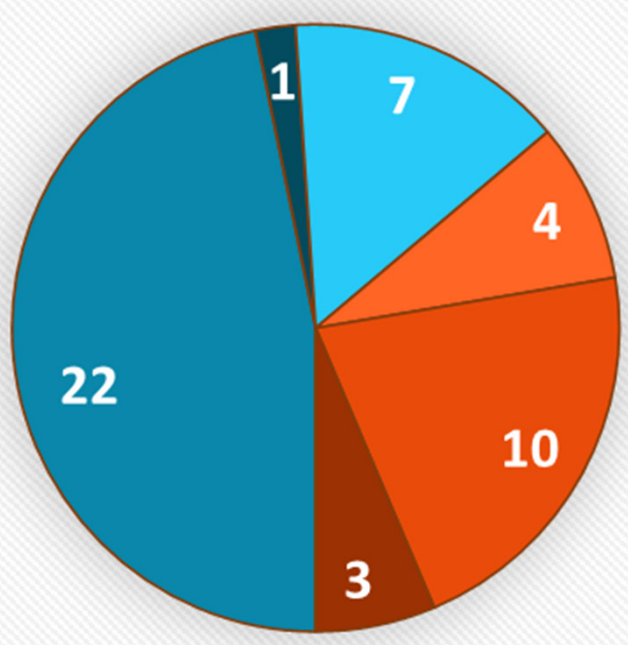

Staphylococcus aureus

Enterococcus faecium

$\square$ Enterobacteriales

$\square$ Streptococcus species

$\square$ CONS

other

Figure 2. Cont. 


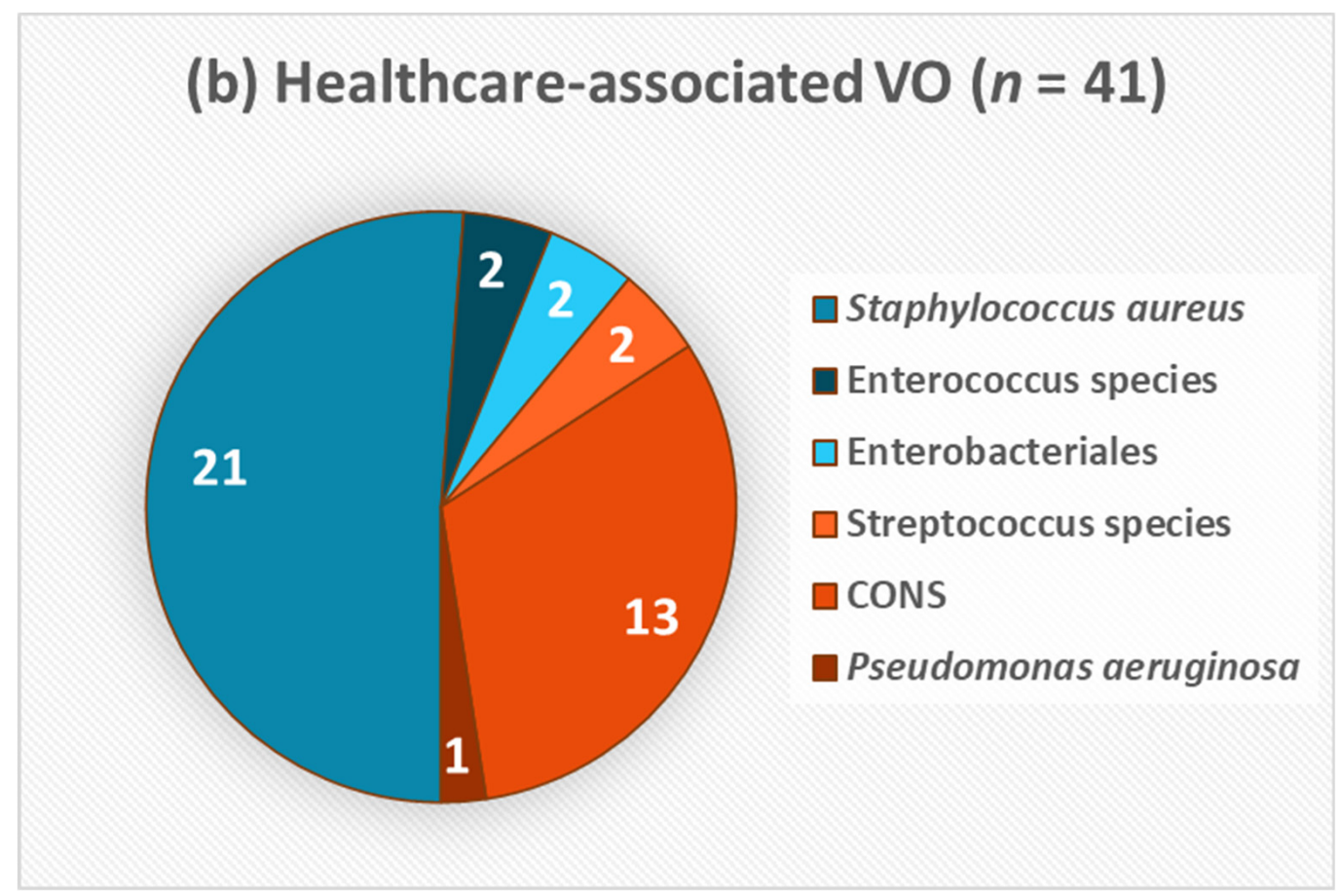

Figure 2. Isolated microorganisms shown in percentage from (a) CAVO and (b) HAVO patients. Other pathogens in CAVO include Propionibacterium acnes, Haemophilus parainfluenzae, and Cutibacterium avidum.

\subsection{Antimicrobial Regimes (3)}

In both the CAVO and HAVO cohort, an antibiotic sensitivity of $100 \%$ could not be achieved with any mono-therapy. Only in the CAVO cohort, $100 \%$ sensitivity could be achieved by the combination of piperacillin-tazobactam and vancomycin or by the combination with meropenem and vancomycin. Figure 3 provides an overview of the predicted efficacy of empiric antimicrobial regimes in both cohorts.

In the HAVO cohort, the highest sensitivity was seen in a mono-therapies with teicoplanin $(90.9 \%)$ and with linezolid $(90.9 \%)$. The combination of meropenem plus vancomycin resulted in a high sensitivity of $95.5 \%$. Both the combinations of vancomycin plus piperacillin-tazobactam or plus ceftriaxone resulted in a sensitivity of $90.9 \%$. The combination of the reserve antibiotics teicoplanin or linezolid with piperacillin-tazobactam or ceftriaxone-cefotaxime-ceftazidim would reach a theoretical sensitivity of $100 \%$ each.

Overall, $82.2 \%$ (HAVO: 68.2\%; CAVO: 95.5\%) of the isolated pathogens were sensitive to piperacillin-tazobactam, $82.2 \%$ (HAVO: $90.9 \%$, CAVO: $73.9 \%$ ) to teicoplanin, $80.0 \%$ (HAVO: $90.9 \%$; CAVO: 69.6\%) to linezolid, $77.8 \%$ (HAVO: $81.8 \%$; CAVO: $73.9 \%$ ) to vancomycin, $64.4 \%$ (HAVO: $68.2 \%$; CAVO: 60.1\%) to clindamycin, and 55.6\% (HAVO: 36.4\%; CAVO: $73.9 \%$ ) to ceftriaxone. Furthermore, $97.8 \%$ of pathogens were sensitive (HAVO: 95.5\%; CAVO: $100.0 \%$ ) to a combination of vancomycin plus meropenem, $91.1 \%$ were sensitive to vancomycin plus co-amoxiclav (HAVO: $86.4 \%$; CAVO: $95.7 \%$ ), $91.1 \%$ were sensitive to vancomycin plus ciprofloxacin (HAVO: $86.4 \%$; CAVO: $95.7 \%$ ), and $93.3 \%$ were sensitive to vancomycin plus cefotaxime (HAVO: 90.9\%; CAVO: 95.7\%). Teicoplanin or Linezolid plus piperacillin-tazobactam would reach a sensitivity of 100.0\% (HAVO: 100.0\%; CAVO: $100 \%)$. The lowest rates of resistance were evident for the combination of meropenem with vancomycin, for which only one strain remained resistant due to an infection with S. epidermidis (HAVO group). Highest overall rates of resistance were found for cefazolin (overall 34.8\% resistant; HAVO: $45.5 \%$; CAVO: 26.1\%) and ciprofloxacin (overall 28.9\% resistant; HAVO: $32.0 \%$; CAVO: $26.1 \%$ ), which could be reduced to $20.0 \%$ (HAVO: $18.2 \%$; CAVO: $21.7 \%$ ) and to $8.9 \%$ (HAVO: $13.6 \%$; CAVO: $4.3 \%$ ), respectively, by an additional combination with vancomycin. 

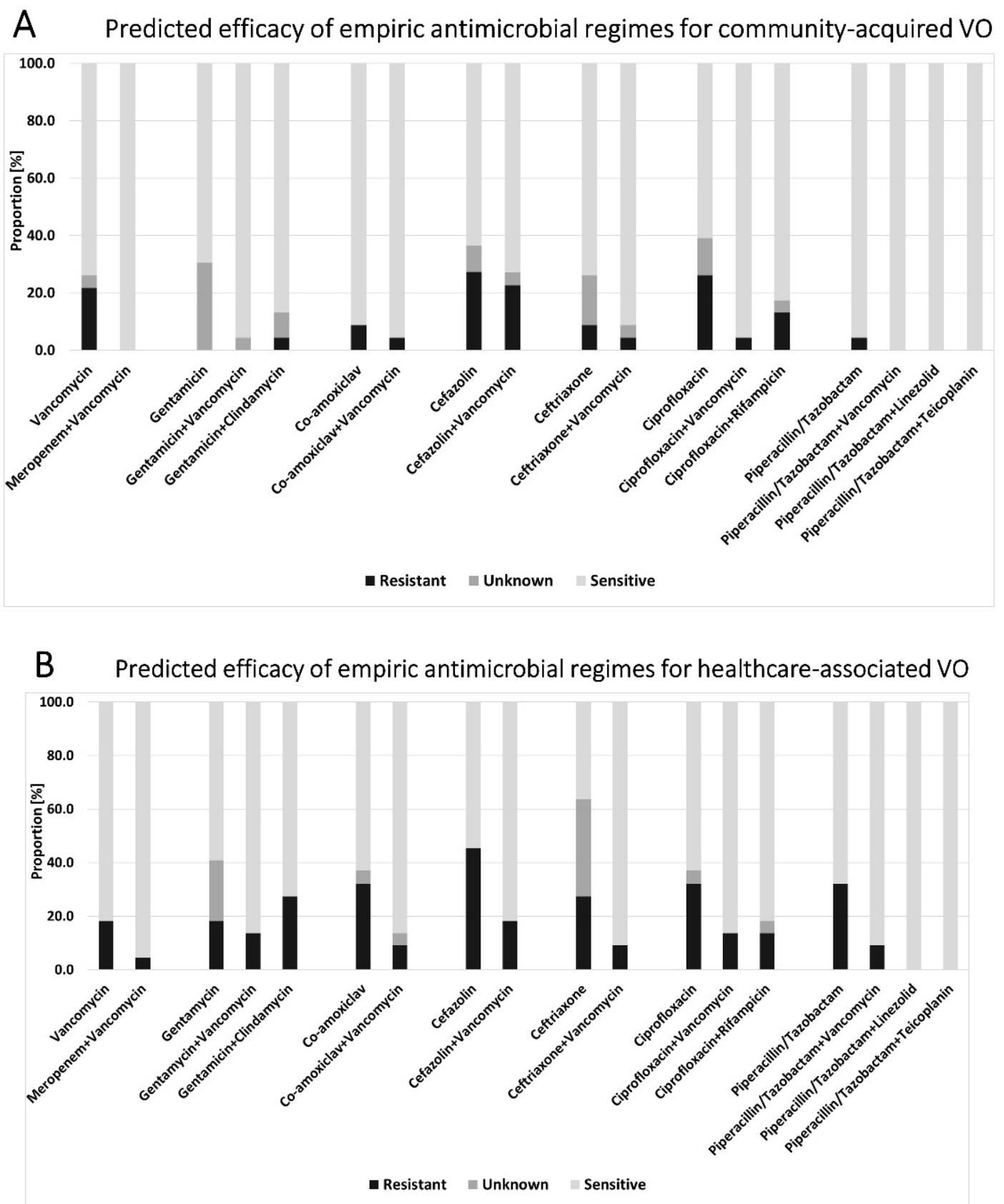

Figure 3. Predicted efficacy of empiric antimicrobial regimes for (A) the CAVO cohort and (B) the HAVO cohort. Bars represent cumulative proportions of resistant and sensitive pathogens and pathogens with unknown sensitivity against the respective agent.

The comparison of Gram-positive- and Gram-negative-stained pathogens showed differences in the distribution of resistances. In Gram-positive pathogens ( $n=37$ cases), the highest sensitivity rates were seen for vancomycin $(94.6 \%)$, linezolid $(97.3 \%)$, and teicoplanin (100\%). The highest resistance rate was documented for ciprofloxacin (29.7\%), followed by meropenem and cefazolin (both $24.3 \%$ ) and piperacillin-tazobactam (21.6\%; Figure 4A). In Gram-negative pathogens ( $n=8$ cases), the highest potential sensitivity was tested for meropenem, imipenem, cotrimoxazole, and piperacillin-tazobactam (each $100.0 \%$ ). Besides vancomycin, linezolid, and teicoplanin, which are not considered suitable for the therapy of Gram-negative infections, the highest resistance rates were seen for cefazolin $(87.5 \%)$ and ciprofloxacin $(75.0 \%$; Figure $4 \mathrm{~B})$. Comparing both groups, apart from vancomycin, linezolid, and teicoplanin (all $p<0.01$ ), the only statistically significant differ- 
ence in the rate of resistance was revealed for the therapy with cotrimoxazole $(U=88.000$; $\mathrm{Z}=-2.146 ; p=0.032$.

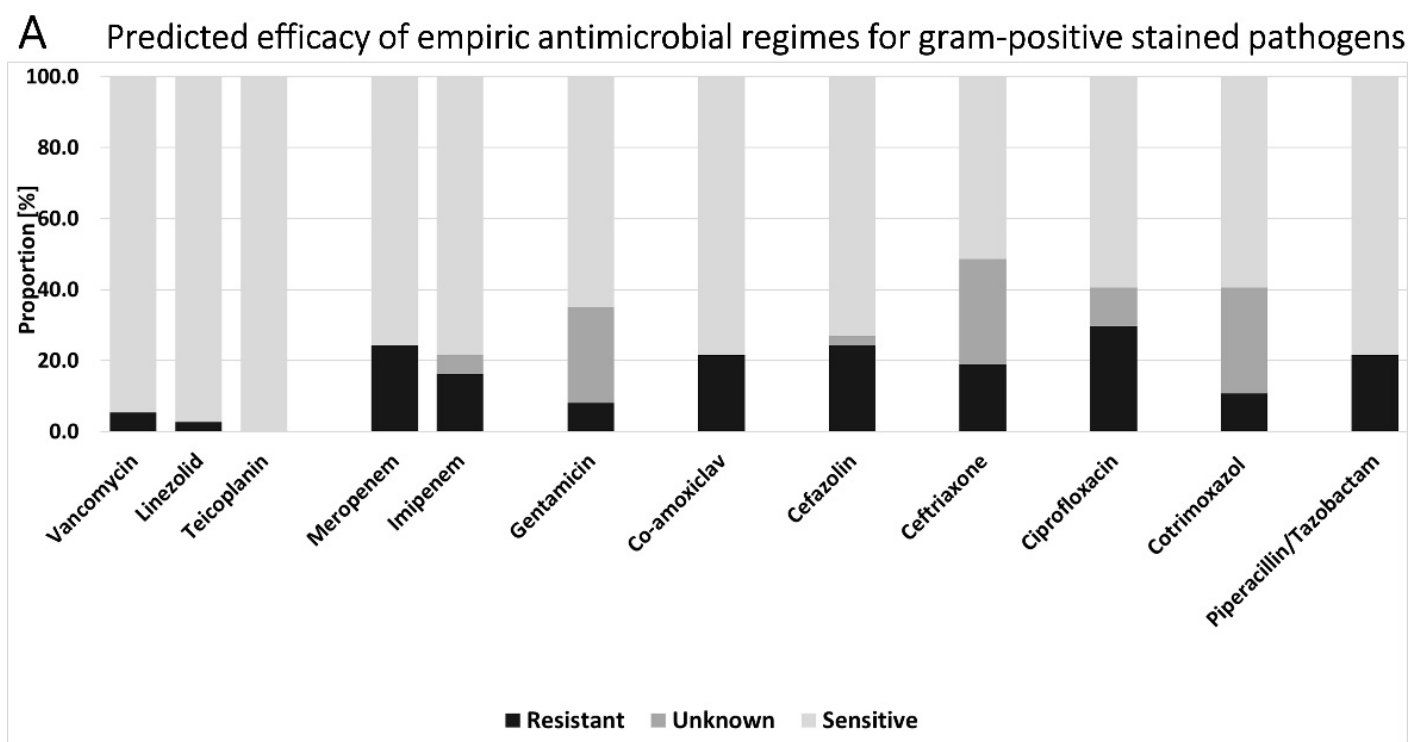

\section{B Predicted efficacy of empiric antimicrobial regimes for gram-negative stained pathogens}

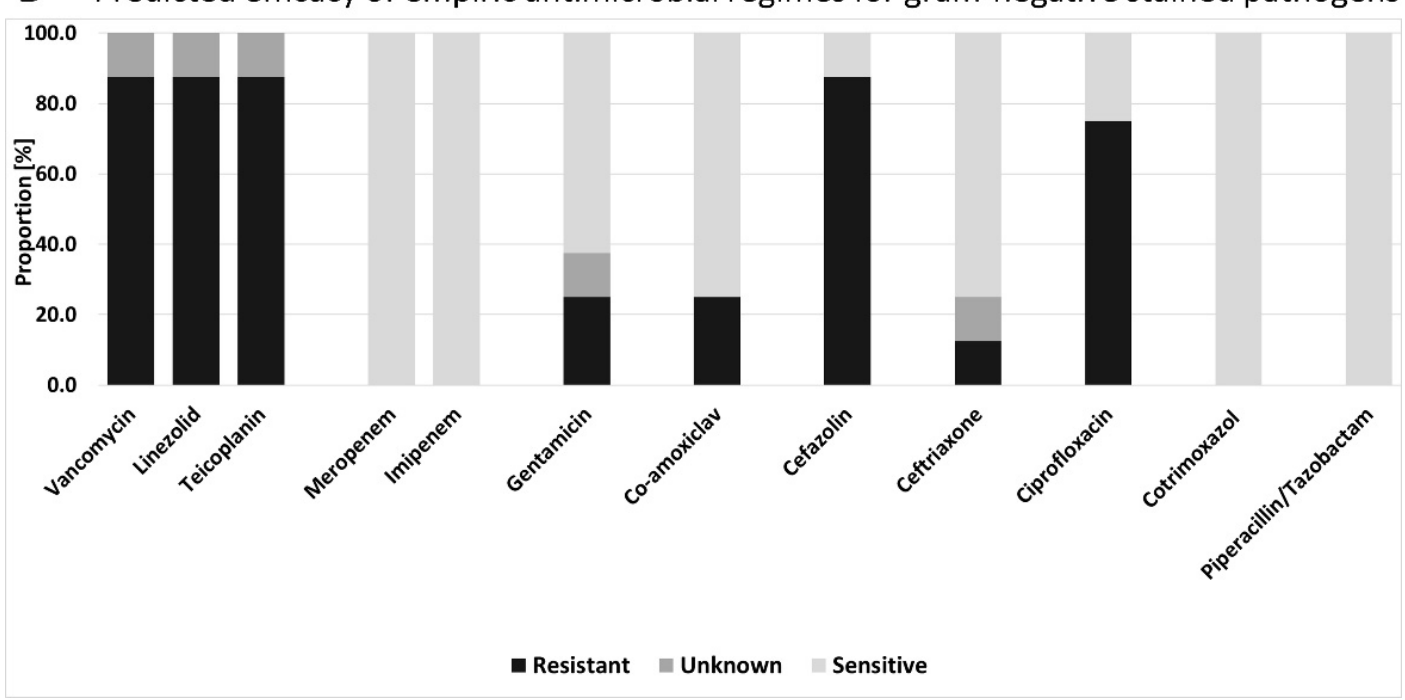

Figure 4. Predicted efficacy of empiric antimicrobial regimes for (A) Gram-positive-stained pathogens $(n=37)$ and (B) Gram-negative-stained pathogens $(n=8)$. Bars represent cumulative proportions of resistant and sensitive pathogens as well as pathogens with unknown sensitivity against the respective agent.

\subsection{Difference of Antibiotic Susceptibility of Pathogens Evidenced in HAVO and CAVO (4)}

Comparing the predicted efficacy of empiric antimicrobial regimens between CAVO and HAVO patients revealed a statistically significant difference regarding the monotherapy with meropenem $(\mathrm{U}=172.000, \mathrm{Z}=-2.654, p=0.008)$, co-amoxiclav $(\mathrm{U}=182.000$, $\mathrm{Z}=-2.232, p=0.026$ ), piperacillin-tazobactam $\mathrm{U}=183.500, \mathrm{Z}=-2.382, p=0.017)$, and ceftriaxone ( $\mathrm{U}=162.000, \mathrm{Z}=-2.304, p=0.021)$. The resistances seen in the HAVO cohort were $36.4 \%$ for meropenem, $31.8 \%$ for co-amoxiclav, $31.8 \%$ for piperacillin-tazobactam, and $27.3 \%$ for ceftriaxone. No statistically significant differences were found between CAVO and HAVO patients regarding tested combined antibiotics (Supplemental Material Table S1). 


\section{Discussion}

The present study compares clinical features and microbiological epidemiology of healthcare-associated and community-acquired vertebral osteomyelitis. Based on antibiotic susceptibility testing of the isolated pathogens, a variety of treatment regimens for empirical antibiotic treatment were analyzed and the most efficient choices were evaluated. The results revealed that underlying pathogens were not statistically significant different for HAVO and CAVO. However, best practice options regarding the sensitivity of antimicrobial regimes were distinct. Antibiotic combinations of either piperacillin-tazobactam + vancomycin or meropenem + vancomycin seem reasonable to achieve a $100 \%$ safe susceptibility to empirical antibiotic therapy for CAVO and approximately $90 \%$ for HAVO.

\subsection{Patient Characteristics and Clinical Presentation and Treatment (1)}

A total of 155 patients with a male to female ratio of 1.3 and a mean age of 66.1 years were included in this study. This is in line with epidemiological studies on VO [1,2,14]. In the current cohort, consisting of $47.7 \% \mathrm{HAVO}$ and $52.3 \%$ CAVO cases, no significant differences in the patient details (age, sex, BMI) or presented symptoms were identified. In contrast, it has been reported that elderly patients had higher rates of healthcare-associated vertebral osteomyelitis than younger patients [15]. Noteworthy, the median CCI was by tendency higher in the HAVO group, and the mean duration of symptoms was around ten days longer compared to the CAVO group. Remarkably, only 25 (16.1\%) patients presented with fever. Despite this, the mean first CRP value was $132.4 \pm 104.1 \mathrm{mg} / \mathrm{L}$ and $n=66$ $(42.6 \%)$ patients had a CRP of $>100 \mathrm{mg} / \mathrm{dL}$. HAVO was associated with a longer duration of symptoms and a longer stay in hospital.

The overall in-hospital mortality rate was $12.9 \%$, without a relevant difference between the groups (CAVO 11.1\%; HAVO 14.9\%). In a current prospective study, Yagdiran reported a 1- and 2-year mortality rate of $20 \%$ and $23 \%$, respectively [16]. They exclusively followed surgically treated patients, whereas in our study, we did not differ between the conservatively and surgically treated patients and only reported on the in-hospital mortality. Pigrau et al. described the risk factors, infectious sources, etiology, clinical features, therapy, and outcome of hematogenous HAVO, and compared the findings with those of CAVO cases in a retrospective cohort study including $41 \mathrm{HAVO}$ cases. They found that, in their setting, one-third of hematogenous pyogenic VO infections were healthcare-associated [9]. Compared with CAVO, the mortality and relapse rates were higher. This is in line with the presented findings that, even though they are not statistically significant. Our findings and the current literature underline that $\mathrm{VO}$, despite advances in treatment options, is still a severe condition associated with high mortality. The influence of a healthcare-associated infection as a potential predictive factor for the survival and clinical outcome remains to be evaluated.

HAVO patients showed a trend of longer duration and longer hospitalization time than CAVO patients, but without statistical significance. Moreover, $63.2 \%$ of patients received an empiric antibiotically treatment, prior to the identification of a pathogen. The empiric antibiosis was found to be inadequate in $42.3 \%$ of cases with an antibiogram. It is crucial that empiric antibiotic should be based on the host and the epidemiologic risk, as well as the local susceptibility patterns. Culture-negative VO is of great importance, in particular. In a retrospective study on 73 patients, Yu et al. recently reported that $\beta$-lactam and glycopeptide antibiotics were mainly used in $\mathrm{VO}$, and fluoroquinolones were used statistically and significantly more in culture-negative $\mathrm{VO}$ [17]. There are limited evidencebased data on national recommendation for the empiric antibiotic regime in VO. There might be considerable differences in daily clinical practice due to a high heterogeneity in local guidelines and experiences that remain to be illuminated more precisely.

\subsection{Aetiology and Microbiological Pattern (2)}

$\mathrm{VO}$ is often difficult to diagnose, especially considering the high rate of patients on antibiotic therapy at the time of diagnosis. This observation results in a low rate of 
positivity from blood cultures and vertebral biopsy to determine the etiology of infection, causing a high rate of culture-negative cases (43.2\%). Even though it is suggested that antibiotic therapy should be withheld until the pathogens are identified, this is often not feasible in clinical practice when patients' conditions are unstable [13].

In a 10-year cohort study, Avenel et al. recently found that, when $\mathrm{VO}$ was suspected on imaging, bacteriological investigation identified the microorganism in 209/300 (70\%) of the cases [18]. The yield of percutaneous needle biopsy was $54.8 \%$ and the only predictor of percutaneous needle biopsy negativity, detected in a multivariate analysis was previous antibiotic intake [18]. There was no statistically significant difference in the frequencies of causative pathogens between the HAVO and CAVO cohort. For both cohorts, S. aureus was the most frequently detected pathogen in both subgroups, which is in line with the literature $[6,17,19]$. The analyzed cohorts did not include polymicrobial infections, which is also in line with other studies reporting low percentages [20]. In the present cohort, $82.2 \%$ of causative pathogens were classified as Gram-positive and $17.8 \%$ as Gram-negative, with no significant differences between the CAVO and HAVO cohort. We found S. aureus to be the most frequent and CoNS to be the second most frequent causative pathogen in the HAVO cohort.

In their study, which included $358 \mathrm{VO}$ cases, Park et al. found that methicillinsusceptible $S$. aureus was the most frequently isolated causative pathogen $(33.5 \%)$, followed by methicillin-resistant S. aureus (MRSA) (24.9\%), Enterobacteriales (19.3\%), and Streptococcus species (11.7\%) [21]. Concordant with our study, they did not find differences in the proportions of pathogens between the HAVO and non-HAVO cohort. They called attention to the higher frequency of MRSA isolates in the HAVO group (43.6\% vs. $13.8 \%$ ), whereas MSSA and Streptococcus spp. were more often isolated in non-HAVO cases [21]. In contrast, we only found one MRSA isolate in the HAVO group but substantial differences in the distribution of methicillin-resistant and healthcare-associated CoNS. Pigrau et al. documented a CoNS infection in $15.0 \%$ in HAVO and of $2.0 \%$ in CAVO cases [9]. A similar trend was seen in our results, which show infections with CoNS in $31.7 \%$ of HAVO and in $21.3 \%$ of CAVO cases. Significantly more methicillin-resistant isolates were found in the HAVO cohort compared to CAVO. In their update on the increasing clinical impact of CoNS infections, Michels et al. point out the extensive antimicrobial resistance profile of CoNS, especially in healthcare settings [22]. Because of the lower virulency of CoNS compared to $S$. aureus in VO [23], these infections may be missed and diagnosed late. Over the past few years, CoNS infections, usually originating from intravascular devices, have been increasingly reported as a cause for hematogenous VO [12,24,25]. Intravascular catheter-associated infections are considered as the sources of healthcare-associated vertebral osteomyelitis in up to $34 \%$ of patients [9]. The higher incidence of antibiotic resistance in causative pathogens, predominantly in CoNS in the current HAVO cohort, must be considered when selecting an empiric antibiotic regimen. We showed that the patients in the HAVO cohort are, by tendency, older with more comorbidities, according to the CCI. Although not directly proven by our results, it can be hypothesized that older and multi-morbid patients are more prone to infections with resistant CoNS, not least because of frequent medical interventions.

\subsection{Empirical Antibiotic Therapy Regimes and Differences between CAVO and HAVO (3 and 4)}

Antibiotic therapy is essential for the treatment of VO. However, guidelines targeting antibiotic treatment strategies are scarce and only limited data are available on the efficacy of antibiotics [26,27]. For the selection of an appropriate empiric antibiotic regime, the local antibiogram profiles should be respected. The highest overall rates of resistance were found for cefazolin (overall 34.8\% resistant; HAVO: 45.5\%; CAVO: $26.1 \%$ ) and ciprofloxacin (overall 28.9\% resistant; HAVO: $32.0 \%$; CAVO: $26.1 \%$ ). A statistically significant difference regarding the mono-therapy between HAVO and CAVO was seen for meropenem, co-amoxiclav, piperacillin-tazobactam and ceftriaxone. Considering the high rates of re- 
sistance for antibiotic mono-therapies both in CAVO and HAVO patients, these must be critically considered in VO.

The main proportion of vancomycin resistance in this study cohort can be lead back to Gram-negative pathogens. Furthermore, a Gram-negative pathogen was only identified in $17.8 \%$ of cases, and there was no significant difference in the distribution between the HAVO and the CAVO cohort. Similarly, Park et al. found a rate of $20.8 \%$ for Gram-negative bacteria in their cohort of 313 microbiologically diagnosed VO cases [28]. By trend, more Gram-negative strains were found in the current CAVO cohort. They showed sensitivities of $100 \%$ for the testes carbapenems, cotrimoxazole, and piperacillin-tazobactam.

Our results indicate a broad coverage for either piperacillin-tazobactam + vancomycin, vancomycin + co/amoxiclav, vancomycin + ciprofloxacin, vancomycin + ceftotaxime, or meropenem + vancomycin, achieving a $95 \%$ safe susceptibility to empirical antibiotic therapy for CAVO and more than $80 \%$ for HAVO. The highest potential coverage in HAVO cases of $95.5 \%$ could be achieved with vancomycin + meropenem. These combinations are most likely to cover Gram-positive and Gram-negative stains. The use of carbabenems should, however, be limited to critically ill patients since the use of carbapenems has been proven to be associated with the risk of acquiring antibiotic-resistant bacteria colonization [29]. A broad-spectrum antibiotic combination encompasses the risk of facilitating antimicrobial resistance. Thus, antimicrobial susceptibility assessment is essential, and initial antibiotic therapy should rapidly be adjusted as soon as pathogens and their antibiograms are identified.

Only a few studies focused on the difference between CAVO and HAVO. Park et al. found that most of the isolated pathogens were susceptible to vancomycin plus ciprofloxacin or broad-spectrum cephalosporin for HAVO [21]. Their findings further suggested that fluoroquinolone-based oral combinations may not be appropriate due to frequent resistance, especially in cases of HAVO [21]. In their cohort, MRSA was the main causative pathogen, whereas in our cohort, only one MRSA case was evident. In contrast, methicillin resistance was mainly found in CoNS isolates in the current study. This difference highlights the importance of the evaluation of local susceptibility patterns.

For critically ill patients and patients with renal dysfunction, the vancomycin dosage must be adapted, and regular control of the serum levels are essential to prevent nephrotoxicity. As alternatives to vancomycin, teicoplanin, linezolid, and daptomycin can be considered. The susceptibility pattern for daptomycin was not routinely tested in our institution; hence, the present study can unfortunately not provide any data on this agent. However, a retrospective analysis of MRSA vertebral osteomyelitis cases showed a higher recurrence rate under vancomycin compared to daptomycin [30]. Linezolid and teicoplanin showed high susceptibility rates (each $90.9 \%$ ) when used as a mono-therapy in HAVO cases but substantial lower rates in CAVO cases (69.6\% and $73.9 \%$, respectively). If combined with piperacillin-tazobactam, linezolid and teicoplanin both showed a theoretical sensitivity of $100 \%$, both in the HAVO and the CAVO cohort. Another feasible approach to bypass unwanted side effects of systemic antibiotics is via the administration of local antibiotic carriers in cases of implant-associated vertebral osteomyelitis [27]. For instance, gentamicin + vancomycin, which should be carefully considered as systemic antibiotic therapy due to nephrotoxicity, are applicable as local antibiotics. Regarding this, Fleege et al. showed a reduction in the duration of systemic antibiotic therapy by the additional use of local antibiotics based on absorbable carrier material in the surgical treatment of bacterial VO [31]. Their study reveals that gentamicin and vancomycin are commonly used agents in local carrier materials. In our cohort, $86.4 \%$ of isolates in the HAVO cohort showed a theoretical susceptibility for a combination of gentamicin + vancomycin, but only $59.1 \%$ of isolates were susceptible to gentamicin alone.

\subsection{Limitations}

This retrospective study has several limitations. Data analysis of one orthopaedictrauma center may lead to a local epidemiological bias. In addition, the retrospective design 
restricts analysis to already-existing resistograms, which were electronically available back to 2013. In some cases, antibiotic testing for certain antibiotics was sometimes not performed, leading to "unknown" listed antibiotic susceptibility. In addition, the retrospective file analysis did not consistently allow identification of antibiotic pretreatment and its effect on the detection of infection-causing pathogens.

\section{Materials and Methods}

\subsection{Patient Identification}

A retrospective cohort study of patients treated for VO was conducted in a level-1 orthopaedic trauma center with a dedicated spine focus in Germany. The inclusion period was defined from 1 January 2000 to 3 December 2020. Eligible cases of patients 18 years or older were screened by international classification of disease (ICD)-10 diagnosis codes (M46.2: osteomyelitis of vertebra; M46.3: infection of the intervertebral disc (pyogenic); M46.4: discitis, unspecified; and M46.5: other infective spondylopathies). Afterwards, patients' medical charts, surgery protocols, laboratory findings, as well as microbiological and histopathological reports were screened for criteria of VO. The diagnosis of VO was confirmed if at least two of the following criteria were documented: Compatible clinical features (1); radiological evidence of vertebral osteomyelitis in CT and/or MRI (2) [32,33]; and microbiologic demonstration of bacterial pathogens, either from the site of infection itself (e.g., abscess, intervertebral disc, or vertebral bone) or in the blood (3) [6,34]. As previously defined by Pigrau et al., HAVO was assumed when at least one of the following criteria were documented [9]:

(i) Onset of symptoms after one month of hospitalization with no evidence of vertebral osteomyelitis at admission;

(ii) Hospital admission within six months before symptom onset;

(iii) Ambulatory diagnostic or therapeutic manipulations within six months before symptom onset (long-term central venous catheter use, arteriovenous fistula for hemodialysis, invasive intravascular techniques, urological, gynecological or digestive procedures, and cutaneous manipulations).

$\mathrm{VO}$ cases that did not meet any of the above criteria were classified as CAVO.

\subsection{Data Collection}

Patient characteristics (sex, age, and BMI at the time of admission), symptoms (backpain, neurological symptoms, fever, and sepsis), clinical features (start of symptoms, hospitalization, treatment modalities, and empiric antibiotics), blood parameters (CRP and leucocyte count), radiological findings (signs of vertebral osteomyelitis in the CT or MRI), and details of VO (spinal height, vertebral levels, and abscess formations (psoas, paravertebral, epidural)) were assessed retrospectively by reviewing electronic medical records. Comorbidities were assessed by obtaining the CCI [35]. The microbiological database was searched for information on the causative pathogens and on their antimicrobial susceptibility testing. Detection was achieved either preoperatively or intraoperatively by deep tissue sampling or aspiration of the affected vertebral segment. Data on antimicrobial susceptibility testing were accessible back to 2013.

\subsection{Microbiology}

Tissue samples were homogenized and seeded on solid and liquid culture media. All samples were incubated for 14 days. Bacteria were identified by matrix-assisted laser desorption ionization time of flight mass spectrometry (MALDI TOF MS) using a Microflex LT mass spectrometer and BioTyper software (Bruker Daltonik, Bremen, Germany). Antibiotic susceptibility testing followed guidelines from the European Committee on Antimicrobial Susceptibility Testing (EUCAST) were applied [36]. Testing was performed using a BD Pheonix M50 nephelometer (Becton, Dickinson and Company, Heidelberg, Germany) or manually by disc diffusion. 


\subsection{Statistics}

Descriptive and statistical data analysis was performed using the IBM SPSS Statistics software (version 28.0, IBM Corp, Armonk, NY, USA). Frequencies were expressed as numbers and percentages. Continuous parameters were presented as means \pm standard deviation (SD) and compared by Student's $t$-test. The Chi-square test was used for comparison of categorical variables. The Mann-Whitney-U-test was used to determine if there were differences in the antimicrobial regimes between CAVO and HAVO patients or between cases with Gram-positive and Gram-negative pathogens. For all tests, $p$ values $<0.05$ were considered statistically significant.

\section{Conclusions}

Healthcare association is common in VO. Antibiotic resistance of CAVO pathogens differed significantly from HAVO. We detected significantly more methicillin-resistant isolates in the HAVO cohort compared to the CAVO cohort. The hypothetical analysis of possible antibiotic regimes reveals that, for an empirical antibiotic therapy, a combination of $\beta$-lactam antibiotics + vancomycin is suitable, both for CAVO and HAVO cases.

Supplementary Materials: The following are available online at https:/ /www.mdpi.com/article/10 .3390 / antibiotics10111410/s1, Table S1: Predicted efficacy of empiric antimicrobial regimes for the CAVO and the HAVO cohort for mono- and combination therapies. Values are numeric and display the relation between the results for CAVO and HAVO cohorts. Statistically significant differences ( $p$-values) are marked in bolt. S: sensitive; R: resistant; $X$ : unknown sensitivity.

Author Contributions: Conceptualization, S.L., M.L., N.W., M.E. and M.R.; methodology, S.L., A.F., V.F., M.E. and M.R.; validation, S.L., C.N., M.L., M.E., V.A. and M.R.; formal analysis, S.L., A.F., V.F., M.E. and M.L.; investigation, S.L., A.F., V.F., N.W. and M.R.; resources, S.L., M.L., C.N., V.A. and M.R., data curation, S.L., A.F., N.W. and M.R.; writing-original draft preparation, S.L., N.W., M.L., M.E., V.A. and M.R.; writing-review and editing, S.L., N.W., V.F., M.L., C.N., M.E., V.A. and M.R.; visualization, S.L., N.W. and M.R.; supervision, S.L., M.L., V.A. and M.R.; project administration, S.L. and M.R.. All authors have read and agreed to the published version of the manuscript.

Funding: This research received no external funding.

Institutional Review Board Statement: This study was carried out in accordance with the Declaration of Helsinki and approved by the ethics committee at the University of Regensburg in 09/2021, 12-218_2-101.

Informed Consent Statement: Informed consent was waived due to the study design. This retrospective register study is based on data from patient charts. No prospective follow-up was conducted, and data were anonymized for the evaluation.

Data Availability Statement: All data presented in this study were available on demand from the corresponding author.

Conflicts of Interest: The authors declare no conflict of interest.

\section{References}

1. Grammatico, L.; Baron, S.; Rusch, E.; Lepage, B.; Surer, N.; Desenclos, J.C.; Besnier, J.M. Epidemiology of vertebral osteomyelitis (VO) in France: Analysis of hospital-discharge data 2002-2003. Epidemiol. Infect. 2008, 136, 653-660. [CrossRef]

2. Fantoni, M.; Trecarichi, E.M.; Rossi, B.; Mazzotta, V.; Di Giacomo, G.; Nasto, L.A.; Di Meco, E.; Pola, E. Epidemiological and clinical features of pyogenic spondylodiscitis. Eur. Rev. Med. Pharmacol. Sci. 2012, 16 (Suppl. S2), 2-7.

3. Jensen, A.; Espersen, F.; Skinhøj, P.; Rosdahl, V.; Frimodt-Møller, N. Increasing frequency of vertebral osteomyelitis following Staphylococcus aureus bacteraemia in Denmark 1980-1990. J. Infect. 1997, 34, 113-118. [CrossRef]

4. Nickerson, E.K.; Sinha, R. Vertebral osteomyelitis in adults: An update. Br. Med. Bull. 2016, 117, 121-138. [CrossRef]

5. Kehrer, M.; Pedersen, C.; Jensen, T.G.; Lassen, A.T. Increasing incidence of pyogenic spondylodiscitis: A 14-year population-based study. J. Infect. 2014, 68, 313-320. [CrossRef] [PubMed]

6. Lang, S.; Rupp, M.; Hanses, F.; Neumann, C.; Loibl, M.; Alt, V. Infections of the spine: Pyogenic spondylodiscitis and implantassociated vertebral osteomyelitis. Unfallchirurg 2021, 124, 489-504. [CrossRef]

7. Hopkinson, N.; Stevenson, J.; Benjamin, S. A case ascertainment study of septic discitis: Clinical, microbiological and radiological features. QJM 2001, 94, 465-470. [CrossRef] 
8. Duarte, R.M.; Vaccaro, A.R. Spinal infection: State of the art and management algorithm. Eur. Spine J. 2013, 22, 2787-2799. [CrossRef] [PubMed]

9. Pigrau, C.; Rodriguez-Pardo, D.; Fernández-Hidalgo, N.; Moretó, L.; Pellise, F.; Larrosa, M.N.; Puig-Asensio, M.; Almirante, B. Health care associated hematogenous pyogenic vertebral osteomyelitis: A severe and potentially preventable infectious disease. Medicine 2015, 94, e365. [CrossRef]

10. McHenry, M.C.; Easley, K.A.; Locker, G.A. Vertebral osteomyelitis: Long-term outcome for 253 patients from 7 Cleveland-area hospitals. Clin. Infect. Dis. Off. Publ. Infect. Dis. Soc. Am. 2002, 34, 1342-1350. [CrossRef]

11. Mylona, E.; Samarkos, M.; Kakalou, E.; Fanourgiakis, P.; Skoutelis, A. Pyogenic vertebral osteomyelitis: A systematic review of clinical characteristics. Semin. Arthritis Rheum. 2009, 39, 10-17. [CrossRef]

12. Renz, N.; Haupenthal, J.; Schuetz, M.A.; Trampuz, A. Hematogenous vertebral osteomyelitis associated with intravascular device-associated infections-A retrospective cohort study. Diagn. Microbiol. Infect. Dis. 2017, 88, 75-81. [CrossRef] [PubMed]

13. Berbari, E.F.; Kanj, S.S.; Kowalski, T.J.; Darouiche, R.O.; Widmer, A.F.; Schmitt, S.K.; Hendershot, E.F.; Holtom, P.D.; Huddleston, P.M., 3rd; Petermann, G.W.; et al. 2015 Infectious Diseases Society of America (IDSA) Clinical Practice Guidelines for the Diagnosis and Treatment of Native Vertebral Osteomyelitis in Adults. Clin. Infect. Dis. Off. Publ. Infect. Dis. Soc. Am. 2015, 61, e26-e46. [CrossRef]

14. Pola, E.; Autore, G.; Formica, V.M.; Pambianco, V.; Colangelo, D.; Cauda, R.; Fantoni, M. New classification for the treatment of pyogenic spondylodiscitis: Validation study on a population of 250 patients with a follow-up of 2 years. Eur. Spine J. 2017, 26, 479-488. [CrossRef] [PubMed]

15. Aguilar-Company, J.; Pigrau, C.; Fernández-Hidalgo, N.; Rodríguez-Pardo, D.; Falcó, V.; Lung, M.; Pellisé, F.; Almirante, B. Native vertebral osteomyelitis in aged patients: Distinctive features. An observational cohort study. Infection 2018, 46, 679-686. [CrossRef] [PubMed]

16. Yagdiran, A.; Otto-Lambertz, C.; Lingscheid, K.M.; Sircar, K.; Samel, C.; Scheyerer, M.J.; Zarghooni, K.; Eysel, P.; Sobottke, R.; Jung, N.; et al. Quality of life and mortality after surgical treatment for vertebral osteomyelitis (VO): A prospective study. Eur. Spine J. 2021, 30, 1721-1731. [CrossRef]

17. Yu, D.; Kim, S.W.; Jeon, I. Antimicrobial therapy and assessing therapeutic response in culture-negative pyogenic vertebral osteomyelitis: A retrospective comparative study with culture-positive pyogenic vertebral osteomyelitis. BMC Infect. Dis. 2020, 20, 939. [CrossRef]

18. Avenel, G.; Guyader, P.; Fiaux, E.; Alcaix, D.; Zarnitsky, C.; Pouplin-Jardin, S.; Kozyreff-Meurice, M.; Lequerré, T.; Vittecoq, O. Microbiological diagnosis of suspected vertebral osteomyelitis with a focus on the yield of percutaneous needle biopsy: A 10-year cohort study. Eur. J. Clin. Microbiol. Infect. Dis. 2021, 40, 297-302. [CrossRef]

19. Gouliouris, T.; Aliyu, S.H.; Brown, N.M. Spondylodiscitis: Update on diagnosis and management. J. Antimicrob. Chemother. 2010, 65 (Suppl. S3), iii11-iii24. [CrossRef]

20. Amsilli, M.; Epaulard, O. How is the microbial diagnosis of bacterial vertebral osteomyelitis performed? An 11-year retrospective study. Eur. J. Clin. Microbiol. Infect. Dis. 2020, 39, 2065-2076. [CrossRef]

21. Park, K.-H.; Kim, D.Y.; Lee, Y.-M.; Lee, M.S.; Kang, K.-C.; Lee, J.-H.; Park, S.Y.; Moon, C.; Chong, Y.P.; Kim, S.-H.; et al. Selection of an appropriate empiric antibiotic regimen in hematogenous vertebral osteomyelitis. PLoS ONE 2019, 14, e0211888. [CrossRef]

22. Michels, R.; Last, K.; Becker, S.L.; Papan, C. Update on Coagulase-Negative Staphylococci-What the Clinician Should Know. Microorganisms 2021, 9, 830. [CrossRef] [PubMed]

23. Lopez, J.; Tatar, Z.; Tournadre, A.; Couderc, M.; Pereira, B.; Soubrier, M.; Dubost, J.-J. Characteristics of spontaneous coagulasenegative staphylococcal spondylodiscitis: A retrospective comparative study versus Staphylococcus aureus spondylodiscitis. BMC Infect. Dis. 2017, 17, 683. [CrossRef] [PubMed]

24. Bucher, E.; Trampuz, A.; Donati, L.; Zimmerli, W. Spondylodiscitis associated with bacteraemia due to coagulase-negative staphylococci. Eur. J. Clin. Microbiol. Infect. Dis. 2000, 19, 118-120. [CrossRef] [PubMed]

25. Schuetz, P.; Mueller, B.; Trampuz, A. Serum procalcitonin for discrimination of blood contamination from bloodstream infection due to coagulase-negative staphylococci. Infection 2007, 35, 352-355. [CrossRef]

26. Oh, W.S.; Moon, C.; Chung, J.W.; Choo, E.J.; Kwak, Y.G.; Kim, S.-H.; Ryu, S.Y.; Park, S.Y.; Kim, B.-N. Antibiotic Treatment of Vertebral Osteomyelitis caused by Methicillin-Susceptible Staphylococcus aureus: A Focus on the Use of Oral $\beta$-lactams. Infect. Chemother. 2019, 51, 284-294. [CrossRef] [PubMed]

27. Fleege, C.; Wichelhaus, T.A.; Rauschmann, M. Systemic and local antibiotic therapy of conservative and operative treatment of spondylodiscitis. Der Orthop. 2012, 41, 727-735. [CrossRef]

28. Park, K.-H.; Cho, O.H.; Jung, M.; Suk, K.-S.; Lee, J.H.; Park, J.S.; Ryu, K.N.; Kim, S.-H.; Lee, S.-O.; Choi, S.-H.; et al. Clinical characteristics and outcomes of hematogenous vertebral osteomyelitis caused by gram-negative bacteria. J. Infect. 2014, 69, 42-50. [CrossRef] [PubMed]

29. Tacconelli, E.; De Angelis, G.; Cataldo, M.A.; Mantengoli, E.; Spanu, T.; Pan, A.; Corti, G.; Radice, A.; Stolzuoli, L.; Antinori, S.; et al. Antibiotic Usage and Risk of Colonization and Infection with Antibiotic-Resistant Bacteria: A Hospital Population-Based Study. Antimicrob. Agents Chemother. 2009, 53, 4264-4269. [CrossRef] [PubMed]

30. Rangaraj, G.; Cleveland, K.; Gelfand, M. Comparative Analysis of Daptomycin and Vancomycin in the Treatment of Vertebral Osteomyelitis. Infect. Dis. Clin. Pract. 2014, 22, 219-222. [CrossRef] 
31. Fleege, C.; Rauschmann, M.; Arabmotlagh, M.; Rickert, M. Development and current use of local antibiotic carriers in spondylodiscitis. Orthopäde 2020, 49, 714-723. [CrossRef] [PubMed]

32. Ledermann, H.P.; Schweitzer, M.E.; Morrison, W.B.; Carrino, J.A. MR Imaging Findings in Spinal Infections: Rules or Myths? Radiology 2003, 228, 506-514. [CrossRef]

33. Jevtic, V. Vertebral infection. Eur. Radiol. Suppl. 2004, 14, E43-E52. [CrossRef] [PubMed]

34. Park, K.H.; Cho, O.H.; Lee, J.H.; Park, J.S.; Ryu, K.N.; Park, S.Y.; Lee, Y.M.; Chong, Y.P.; Kim, S.H.; Lee, S.O.; et al. Optimal Duration of Antibiotic Therapy in Patients with Hematogenous Vertebral Osteomyelitis at Low Risk and High Risk of Recurrence. Clin. Infect. Dis. 2016, 62, 1262-1269. [CrossRef]

35. Charlson, M.E.; Pompei, P.; Ales, K.L.; MacKenzie, C.R. A new method of classifying prognostic comorbidity in longitudinal studies: Development and validation. J. Chronic. Dis. 1987, 40, 373-383. [CrossRef]

36. EUCAST: AST of Bacteria n.d. Available online: https:/ / www.eucast.org/ast_of_bacteria/ (accessed on 8 November 2021). 